\title{
Target Detection Method for Intensity VHF Wavelength-Resolution SAR Images
}

\author{
Gustavo H. M. Voigt, Dimas I. Alves, Crístian Müller, Renato Machado, Viet T. Vu, and Mats I. Pettersson
}

\begin{abstract}
This paper presents a detection method for intensity very-high frequency (VHF) wavelength-resolution synthetic aperture radar (SAR) images. The method is based on the likelihoodratio test, considering a bivariate exponential distribution for the tested hypothesis. A common image is used for obtaining two difference images, one considering a surveillance image and another, a reference image. The method uses the difference images to reduce the number of false alarms related to strong scatterers not related to targets. The method is assessed through received operational curves with VHF ultrawideband SAR images obtained by the CARABAS II system. The experimental results reveal the potential of the proposed approach compared with other detection methods.
\end{abstract}

Keywords-ROC, CARABAS II, Likelihood-ratio Test, VHF UWB SAR.

\section{INTRODUCTION}

Historically, change detection methods have been used in multiples applications for synthetic aperture radar (SAR) images [1], such as target detecting or for monitoring an area of interest. Foliage-Penetrating (FOPEN) SAR systems provide images that can be used to detect concealed targets in regions with high-density vegetation [2]. These images generally are obtained using ultra-wideband (UWB) radars operating on very-high frequency (VHF) or ultra-high frequency (UHF) frequency bands. These systems are characterized by resolution in the order of the radar signal wavelengths and are often referred to as wavelength resolution SAR systems [3], [4].

This characteristic gives the wavelength-resolution SAR systems immunity to the speckle noise, which is one of the main problems related to SAR images. Additionally, because the wavelengths in VHF frequency are in the order of a few meters, small scatterers on the ground area of interest are not detected. On the other hand, large scatterers tend to be stable in time and less influenced by environmental effects [5], [6]. Therefore, wavelength resolution SAR systems can obtain images with high temporal stability of the same ground area, making it possible to perform change detection (CD) methods without using clutter reduction techniques.

Change detection methods using amplitude SAR images for wavelength resolution systems have been widely discussed [7] - [9]. Those methods present high performance in terms of the probability of detection and false alarm

Gustavo Henrique Mittmann Voigt, Dimas Irion Alves, and Crístian Müller Federal University of Pampa (UNIPAMPA), Alegrete - RS, Brazil. Email: (gustavovoigt.aluno, dimasalves, cristianmuller)@unipampa.edu.br. Viet T. Vu, and Mats I. Pettersson, Blekinge Institute of Technology (BTH), Karlskrona, Sweden. Email: (viet.thuy.vu, mats.pettersson)@bth.se; Renato Machado, Aeronautics Institute of Technology (ITA), São José dos Campos, Brazil. Email: rmachado@ita.br. rate (FAR) [7] - [11]. Methods recently published explore different strategies to obtain performance gains, such as using a small stack of images [12], machine learning and neural networks [13], or Bayes theorem [14]. Thus, change detection methods for wavelength-resolution SAR images is a research topic of interest. For traditional microwave SAR images, there are many change detection contributions to intensity images [15], [16]. However, to the best of the authors' knowledge, there are only a few studies on change detection methods for intensity VHF UWB SAR images.

Intensity images highlight the contribution of highamplitude pixels, which are related to the presence of strong scatterers. As a result, generally, CD methods provide high detection probability performance. However, the number of false alarms tends to be high too. To reduce the influence of the strong scatterers that are not related to targets, we propose a change detection scheme using three images as input. An incommon image is used for obtaining two difference images, one considering a surveillance image and the other, a reference image. A similar approach was first presented in [17]. However, no performance evaluation was presented.

The proposed detection method is a variation of the CD method presented in [17]. Both methods use the bivariate exponential distribution on a likelihood ratio test (LRT) instead of other more complex distributions such as Gaussian or Gamma used on traditional amplitude images. The proposed $\mathrm{CD}$ method is assessed considering the data set obtained by the UWB VHF SAR CARABAS II system. Receiver operating characteristic (ROC) curves are presented, and the performance of the proposed method is compared with other CD methods.

The contributions of this paper are the following:

- Variation of the proposed method in [17], where only changes associate with positive-amplitude pixels are detected;

- Evaluation of the proposed method considering the entire available image data set since only a few image pairs were considered in [17]. The methods are assessed in terms of probability of detection and false alarm rate (ROC curves);

- The statistical analysis of the proposed target detection method is provided.

The following sections are organized as follows. Section II presents the database considered in this letter. Section III provides the statistical analysis of the target detection method described in this paper. Section IV presents the simulation results and some discussions. Finally, Section V presents some concluding remarks. 


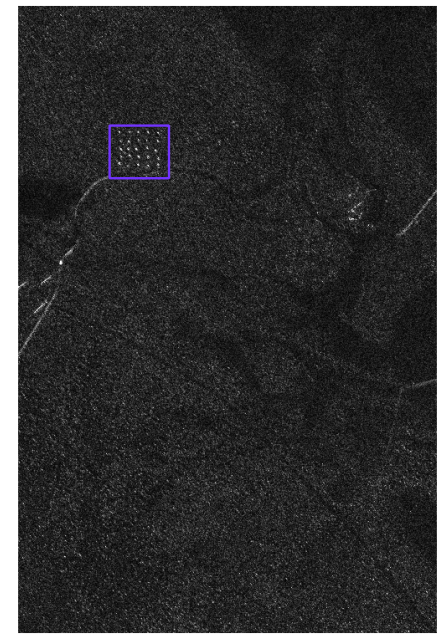

(a)

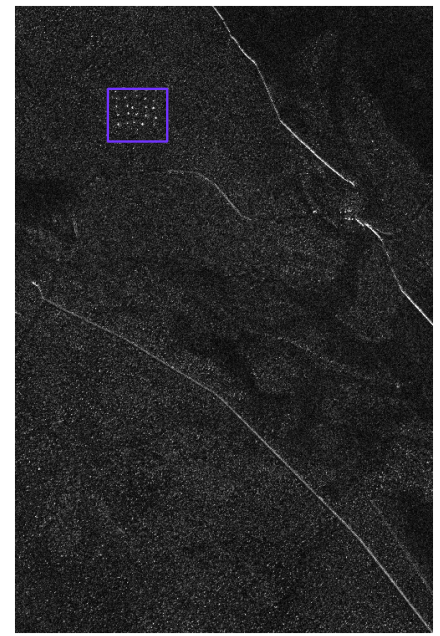

(b)

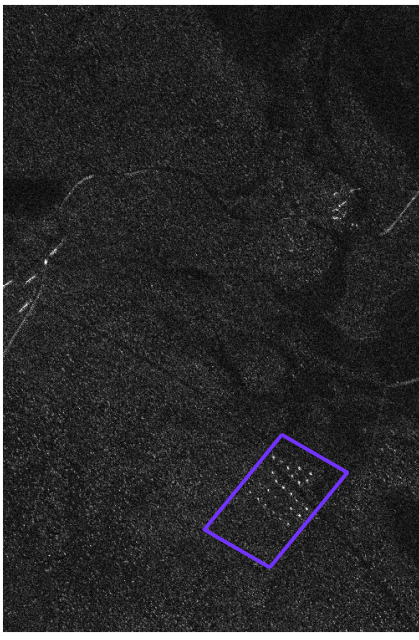

(c)

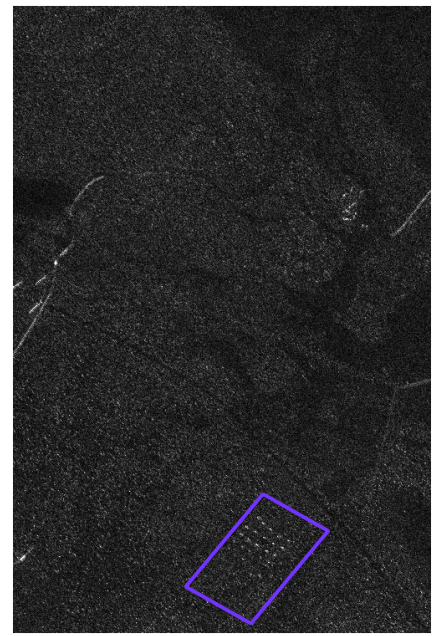

(d)

Fig. 1. CARABAS II image samples for (a) Mission 2 and Pass 1 (b) Mission 3 and Pass 2 (c) Mission 4 and Pass 5 (d) Mission 5 and Pass 1 . The target deployments of each image are highlighted.

\section{DATA DESCRIPTION}

This data set is formed by 24 VHF UWB incoherent wavelength-resolution SAR images with a resolution cell of $3 \mathrm{~m} \times 3 \mathrm{~m}$. The images were obtained from flight campaigns over the same ground area in northern Sweden, covering $2 \mathrm{~km} \times 3 \mathrm{~km}$ with a pixel's size of $1 \mathrm{~m} \times 1 \mathrm{~m}$ [18].

For organization purposes, the 24 images were divided according to four different target deployments (missions) and six different flight scenarios (passes). Each image contains 25 targets, which are terrestrial vehicles. The targets can be divided into ten "small" size vehicles, eight "medium" size vehicles, and seven "large" size vehicles [8]. Also, the data set are already calibrated and geocoded. One example of each target deployment is presented in Figure 1, where the target area is highlighted. Finally, for the sake of simplicity, it is considered the same image classification as the one used in FOI challenge paper [8].

\section{TARGET DETECTION ANALYSIS}

The proposed target detection method for intensity SAR images is based on a hypothesis test to determine if a target is present or absent in a specific pixel position. Using the same problem formulation as [2], [8] and [9], the considered problem can be expressed as

$$
\begin{aligned}
& H_{0}: z=q \quad \text { (no target), } \\
& H_{1}: z=s+q \quad \text { (target), }
\end{aligned}
$$

where $H_{0}$ is the hypothesis that the evaluated pixel is background-related, and $H_{1}$ is the hypothesis that the evaluated pixel is target-related (change), $s$ is related to the target, and $q=c+n$, in which $c$ is related to the clutter, and $n$ to the noise. A classical approach to solve this problem is considering the use of a likelihood-ratio test.

\section{A. Likelihood-ratio Test (LRT)}

As previously mentioned, the proposed target detection method for VHF UWB SAR intensity images is based on an LRT using the bivariate exponential distribution. The input information comes from three images, where one is used as in common image to the surveillance and reference images. The selection of the exponential distribution was based on the nature of SAR images and knowing that the SAR intensity images are frequently modeled using the exponential distribution [19]. The LRT can be written as

$$
\Lambda(z)=\frac{P\left(z \mid H_{1}\right)}{P\left(z \mid H_{0}\right)}
$$

where both $P\left(z \mid H_{1}\right)$ and $P\left(z \mid H_{0}\right)$ statistics are modeled using a bivariate exponential distribution. According to [20], the probability density function (PDF) of the bivariate exponential distribution can be written as

$$
f(x, y)=\frac{\mu_{1} \mu_{2}}{1-\rho} \exp \left[-\frac{\mu_{1} x+\mu_{2} y}{1-\rho}\right] \text { Io }\left[\frac{2 \sqrt{\rho \mu_{1} \mu_{2} x y}}{1-\rho}\right],
$$

where $\mu_{1}$ and $\mu_{2}$ are defined as the inverse of the expectations of $X$ and $Y$, respectively, $\rho$ is defined as the correlation coefficient between $X$ and $Y$, and $I_{o}$ is defined as the modified Bessel function of the first kind of order zero.

Based on the hypotheses in (1) and (2) and the bivariate exponential distribution in (3), it is possible to write the hypotheses probabilities $P\left(z \mid H_{0}\right)$ and $P\left(z \mid H_{1}\right)$, respectively, as

$P\left[z \mid H_{0}\right]=\frac{\mu_{1} \mu_{2}}{1-\rho} \exp \left[-\frac{\mu_{1} x^{2}+\mu_{2} y^{2}}{1-\rho}\right]$ Io $\left[\frac{2 \sqrt{\rho \mu_{1} \mu_{2} x^{2} y^{2}}}{1-\rho}\right]$, 


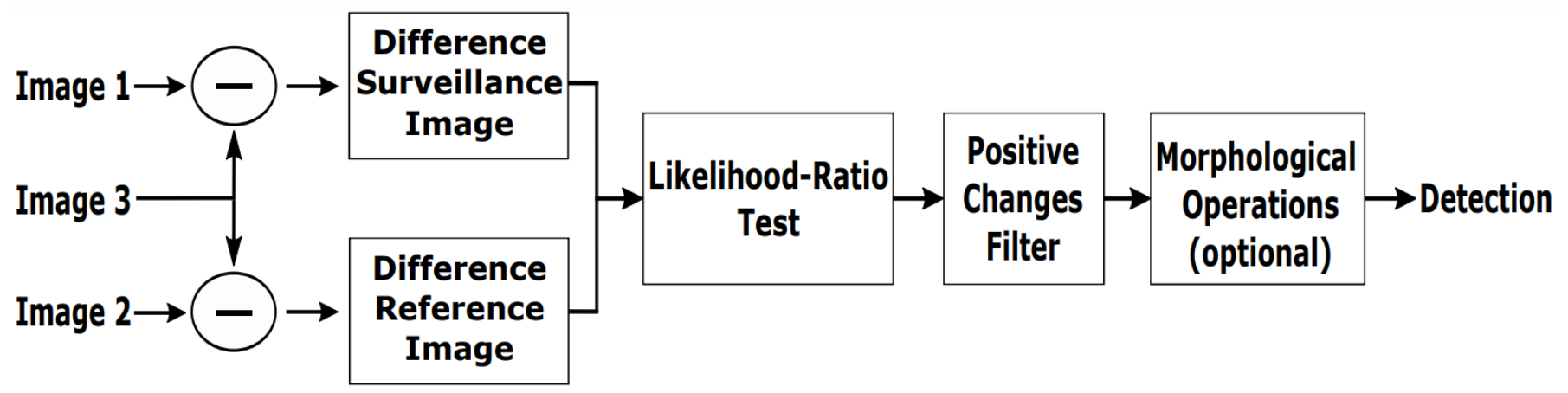

Fig. 2. Block diagram of the proposed change detection method.

$$
\begin{array}{r}
P\left[z \mid H_{1}\right]=\frac{\mu_{1} \mu_{2}}{1-\rho} \exp \left[-\frac{\mu_{1}\left(x-S_{X}\right)^{2}+\mu_{2}\left(y-S_{Y}\right)^{2}}{1-\rho}\right] \\
\text { Io }\left[\frac{2 \sqrt{\rho \mu_{1} \mu_{2}\left(x-S_{X}\right)^{2}\left(y-S_{Y}\right)^{2}}}{1-\rho}\right],
\end{array}
$$

where $z=(x, y), x$ and $y$ are the pixel intensity in the evaluated position, respectively, in the reference and surveillance images. Under the assumption that no targets are observed in the reference image, it is possible to consider $S_{X}$. Thus, in the similar way as presented in [17], the LRT can be written as

$$
\frac{P\left[z \mid H_{1}\right]}{P\left[z \mid H_{0}\right]}=\exp \left[\frac{\mu_{2} S_{Y}\left(2 y-S_{Y}\right)}{1-\rho}\right] \frac{\text { Io }\left[\frac{2 \sqrt{\rho \mu_{1} \mu_{2} x^{2}\left(y-S_{Y}\right)^{2}}}{1-\rho}\right]}{\operatorname{Io}\left[\frac{2 \sqrt{\rho \mu_{1} \mu_{2} x^{2} y^{2}}}{1-\rho}\right]},
$$

where $S_{Y}$ was substituted by $s$ throughout the rest of this paper for the sake of simplicity.

\section{B. Target Detection Method}

A simplified block diagram for the target detection method presented in this paper is described in Figure 2. The block diagram is formed by four basics steps. First, the surveillance and reference difference images are formed. Then, it follows the Likelihood-ratio test that has been described previously. After that, a filter to detect only the positive changes is performed. Finally, there is an optional block of morphological operations (we consider morphological operations in this paper, but this is optional for the method).

The formation of surveillance and reference difference images is performed by a simple subtraction operation. For the surveillance difference image, the input is the difference between Image 1 and Image 3 (see Figure 2). For the reference difference image, the input is the difference between Image 2 and Image 3 (see Figure 2). After that step, both images are changed from amplitude to intensity images by using the operation $|\cdot|^{2}$ in the entire image.

The method can detect targets in the surveillance image associated with both positive and negative amplitude pixels. However, looking to a fair comparison with other notorious methods, we decide to analyze changes only associated with positive-amplitude pixels. We have used a filter to ignore changes associated with negative pixels.

The likelihood-ratio test, previously described in (4), performs a thresholding operation that consists of assigning 1 when $\Lambda(z) \geq T h$ and assigning 0 when $\Lambda(z)<T h$. These block results in a binary data matrix. As in [8], a window of $100 \times 100$ pixels with a step of 10 pixels was used to obtain the statistics considered in (4). The output of this block is a binary image where morphological operations may be applied.

\section{EXPERIMENTAL RESULTS}

The experimental tests were performed considering the proposed target detection method and using all the 24 images provided in the CARABAS-II dataset. The proposed method is evaluated in terms of detection probability $\mathrm{Pb}$, i.e., the number of false alarms per kilometer square. In our evaluation, detection is defined as any detected object in the output binary matrix after morphological operations. Thus, correct target detection is defined as any detected object lying in a range of a maximum of $10 \mathrm{~m}$ from the original target position. Similarly, a false alarm is defined as any detected object that is not considered a target.

Regarding the implementation aspects, the intensity constant $s$ and the threshold have been empirically defined for this initial study, resulting in obtaining multiple ROC curves. Similar morphological operations considered in [8]-[9] were used to perform a fair comparison. The first morphological operation used is erosion with the size of the system resolution cell $(3 \mathrm{~m} \times 3 \mathrm{~m})$. For the dilation, it is used square structuring elements whose sizes enable merging any detected samples that are separated by up to ten meters.

As an initial investigation of the proposed detection method performance, Table I presents the formation of the difference image pairs considered throughout this paper. For simplicity, the name of the images has been shorted using the notation where $\mathrm{Mx}$ represents mission $\mathrm{x}$, and Py represents pass $\mathrm{y}$. As can be noted, experiment 18 has the lowest performance when comparing with the others. This behavior was observed and discussed before [21]. To obtain better performance for this specific experiment, additional processing is possibly required [3]. Finally, it is important to highlight that this outlier tends to degrade the general FAR performance of the proposed method. 
TABLE I

PERFORMANCE RESUlTS OF THE PROPOSED METHOD FOR A THRESHOLD $T h=0.5$ AND $s=0.275$.

\begin{tabular}{|c|c|c|c|c|c|c|c|c|}
\hline \multirow[t]{2}{*}{ Experiments } & \multicolumn{2}{|c|}{$\begin{array}{l}\text { Surveillance } \\
\text { Image }\end{array}$} & \multicolumn{2}{|c|}{$\begin{array}{l}\text { Reference } \\
\text { Image }\end{array}$} & \multirow{2}{*}{$\begin{array}{c}\text { Detected } \\
\text { Targets }\end{array}$} & \multirow{2}{*}{$\begin{array}{l}\text { Probability of } \\
\text { Detection }\end{array}$} & \multirow{2}{*}{$\begin{array}{l}\text { False } \\
\text { Alarm }\end{array}$} & \multirow{2}{*}{$\begin{array}{l}\text { False Alarm } \\
\text { Rate }\left(\mathrm{km}^{-2}\right)\end{array}$} \\
\hline & Image A & Image $\mathrm{C}$ & Image B & Image $\mathrm{C}$ & & & & \\
\hline 1 & M2P1 & M3P1 & M4P1 & M3P1 & 25 & 1 & 1 & 0.16 \\
\hline 2 & M3P1 & M4P1 & M5P1 & M4P1 & 25 & 1 & 5 & 0.83 \\
\hline 3 & M4P1 & M5P1 & M2P1 & M5P1 & 25 & 1 & 0 & 0 \\
\hline 4 & M5P1 & M2P1 & M3P1 & M2P1 & 25 & 1 & 4 & 0.66 \\
\hline 5 & M2P2 & M3P2 & M4P2 & M3P2 & 25 & 1 & 1 & 0.16 \\
\hline 6 & M3P2 & M4P2 & M5P2 & M4P2 & 25 & 1 & 2 & 0.33 \\
\hline 7 & M4P2 & M5P2 & M2P2 & M5P2 & 25 & 1 & 5 & 0.83 \\
\hline 8 & M5P2 & M2P2 & M3P2 & M2P2 & 24 & 0.96 & 0 & 0 \\
\hline 9 & M2P3 & M3P3 & M4P3 & M3P3 & 25 & 1 & 3 & 0.5 \\
\hline 10 & M3P3 & M4P3 & M5P3 & M4P3 & 25 & 1 & 0 & 0 \\
\hline 11 & M4P3 & M5P3 & M2P3 & M4P3 & 25 & 1 & 2 & 0.33 \\
\hline 12 & M5P3 & M2P3 & M3P3 & M2P3 & 25 & 1 & 4 & 0.66 \\
\hline 13 & $\mathrm{M} 2 \mathrm{P} 4$ & M3P4 & M4P4 & M3P4 & 25 & 1 & 2 & 0.33 \\
\hline 14 & M3P4 & M4P4 & M5P4 & M4P4 & 25 & 1 & 1 & 0.16 \\
\hline 15 & M4P4 & M5P4 & M2P4 & M5P4 & 25 & 1 & 3 & 0.5 \\
\hline 16 & M5P4 & M2P4 & M3P4 & M2P4 & 23 & 0.92 & 2 & 0.33 \\
\hline 17 & M2P5 & M3P5 & M4P5 & M3P5 & 25 & 1 & 1 & 0.33 \\
\hline 18 & M3P5 & M4P5 & M5P5 & M4P5 & 22 & 0.88 & 147 & 24.5 \\
\hline 19 & M4P5 & M5P5 & M2P5 & M5P5 & 25 & 1 & 2 & 0.33 \\
\hline 20 & M5P5 & M2P5 & M3P5 & M2P5 & 25 & 1 & 7 & 1.16 \\
\hline 21 & M2P6 & M3P6 & M4P6 & M3P6 & 25 & 1 & 0 & 0 \\
\hline 22 & M3P6 & M4P6 & M5P6 & M4P6 & 25 & 1 & 1 & 0.16 \\
\hline 23 & M4P6 & M5P6 & M2P6 & M5P6 & 25 & 1 & 4 & 0.66 \\
\hline 24 & M5P6 & M2P6 & M3P6 & M2P6 & 25 & 1 & 1 & 0.16 \\
\hline & & Total & & & 594 & 0.99 & 199 & 0.98 \\
\hline
\end{tabular}

The second performance evaluation is presented in Figure 3. We considered different $s$ values and the effect in the ROC curve. For a FAR $=10^{\circ}$, the following probabilities of detection are observed: $P d=96.1 \%$ for $s=0.2, P d=98.1 \%$ for $s=0.25$ and $P d=98.3 \%$ for $s=0.3$. No results were obtained for $s=0.35$ with this FAR, for the evaluated thresholds. Note that increasing $s$, the probability of detection is improved, but the total number of detection is reduced, i.e., the detection saturates earlier. Also, we can observe that for $s=0.35$, we obtain the best Pd with low levels of FAR, but morphological operations eliminate some targets. Thus, lower values of $s$ could be adopted to characterizing targetlike patterns in this data set. Optimal values of this parameter will be the theme for further study.

The third performance evaluation is the ROC curve presented in Figure 4. Here we are interested in comparing the proposed intensity target detection method and others based on amplitude images. The method presented in [7] has, to the best of the author's knowledge, the best performance for this data set adopting the same constraints. The notation used in the originals papers was kept. Analyzing the results, for a $\mathrm{FAR}=10^{0}$, the following probabilities of detection are observable:

- For the reference method: $P d=97.6 \%$ for $s_{1}=0.4$ and
$P d=97.8 \%$ for $s_{1}=0.3$, where $s_{1}$ respects reference paper nomenclature.

- For the proposed method: $P d=98.1 \%$ for $s=0.25$, no results are achieved for $s=0.35$ in this exact FAR, for the evaluated thresholds, but as mentioned before, when adopting this $s$, we obtain the best Pd with lower levels of FAR.

As a result, the proposed method can reach higher $P d$ for lower FAR. The proposed method is similar to the reference method for lower $P d$ (usually not interesting for application purposes).

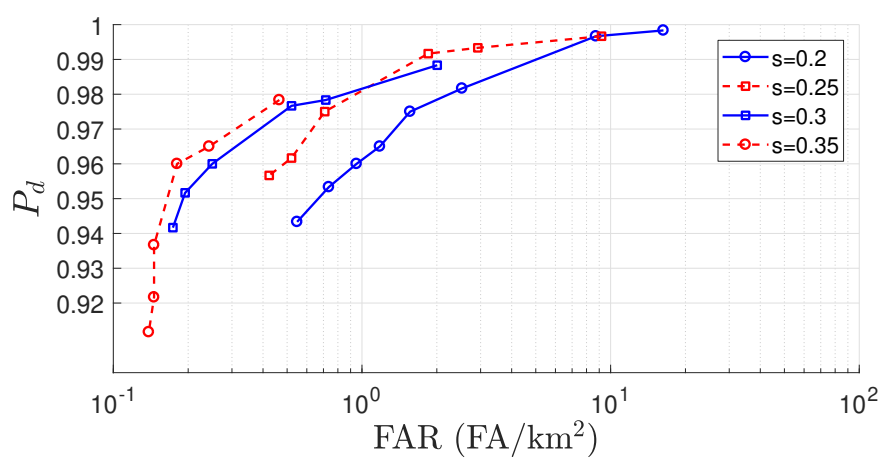

Fig. 3. ROC performance of the proposed method, varying the " $s$ " value. 


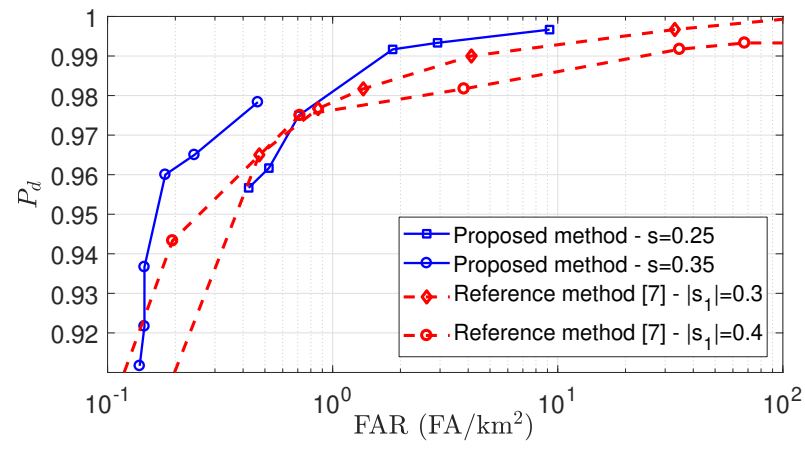

Fig. 4. ROC performance for the proposed and reference [7] methods.

\section{CONCLUSION}

The proposed target detection method for intensity VHF wavelength-resolution SAR images has a competitive performance compared with other detection methods for the evaluated metrics. Additionally, since this paper is an initial study, no investigation was presented regarding the optimization of the intensity constant, threshold, and morphological operations. Nevertheless, better performance was achieved using a clutter distribution with a better fit between data and the statistical model. The investigation of the mentioned parameters and better-fitted distributions are topics for future studies.

\section{ACKNOWLEDGMENT}

This work was supported in part by the Brazilian Agencies National Council for Scientific and Technological Development $(\mathrm{CNPq})$, by Saab AB, and Coordination for the Improvement of Higher Education Personnel (CAPES) - Finance Code 001 (Pró-Defesa IV).

\section{REFERENCES}

[1] M. A. Richards, J. Scheer, W. A. Holm, and W. L. Melvin, Principles of modern radar, 1st ed. Citeseer, 2010.

[2] L. M. H. Ulander, W. E. Pierson, M. Lundberg, P. Follo, P.-O. Frolind, and A. Gustavsson, "Performance of VHF-band SAR change detection for wide-area surveillance of concealed ground targets," in SPIE Defense and Security Symposium: Algorithms for Synthetic Aperture Radar Imagery XI, vol. 5427, 2004.

[3] V. T. Vu, M. I. Pettersson, R. Machado, P. Dammert, and H. Hellsten, "False alarm reduction in wavelength-resolution sar change detection using adaptive noise canceler," IEEE Transactions on Geoscience and Remote Sensing, vol. 55, no. 1, pp. 591-599, Jan. 2017.

[4] P.-O. Frolind and L. Ulander, "Motion compensation effects for repeatpass processing in wavelength-resolution SAR," in IGARSS '98. Sensing and Managing the Environment. IEEE International Geoscience and Remote Sensing. Symposium Proceedings. (Cat. No. 98CH36174), vol. 5, Jul. 1998, pp. 2637-2639.
[5] R. Machado, V. T. Vu, M. I. Pettersson, P. Dammert, and H. Hellsten, "The stability of UWB low-frequency SAR images," IEEE Geoscience and Remote Sensing Letters, vol. 13, no. 8, pp. 1114-1118, Aug. 2016.

[6] G. Smith and L. Ulander, "A model relating VHF-band backscatter to stem volume of coniferous boreal forest," IEEE Transactions on Geoscience and Remote Sensing, vol. 38, no. 2, pp. 728-740, Mar. 2000.

[7] V. T. Vu, N. R. Gomes, M. I. Pettersson, P. Dammert, and H. Hellsten, "Bivariate Gamma distribution for wavelength-resolution SAR change detection," IEEE Transactions on Geoscience and Remote Sensing, vol. 57, no. 1, pp. 473-481, Jan. 2019.

[8] M. Lundberg, L. M. H. Ulander, W. E. Pierson, and A. Gustavsson, "A challenge problem for detection of targets in foliage," in SPIE Defense and Security Symposium: Algorithms for Synthetic Aperture Radar Imagery XIII, vol. 6237, May 2006.

[9] L. M. H. Ulander, M. Lundberg, W. Pierson, and A. Gustavsson, "Change detection for low-frequency SAR ground surveillance," IEE Proceedings - Radar, Sonar and Navigation, vol. 152, no. 6, pp. 413420, Dec. 2005.

[10] L. P. Ramos, A. B. Campos, C. Schwartz, L. T. Duarte, D. I. Alves, M. I. Pettersson, V. T. Vu, and R. Machado, "A wavelength-resolution sar change detection method based on image stack through robust principal component analysis," Remote Sensing, vol. 13, no. 5, 2021. [Online]. Available: https://www.mdpi.com/2072-4292/13/5/833

[11] B. G. Palm, D. I. Alves, M. I. Pettersson, V. T. Vu, R. Machado, R. J. Cintra, F. M. Bayer, P. Dammert, and H. Hellsten, "Wavelengthresolution SAR ground scene prediction based on image stack," Sensors, vol. 20, no. 7, Apr. 2020.

[12] V. T. Vu, D. I. Alves, B. G. Palm, M. I. Pettersson, P. Dammert, and H. Hellsten, "A detector for wavelength resolution SAR incoherent change detection," in IEEE Radar Conference (RadarConf), Sep. 2019, pp. 1-5.

[13] S. Yang, Z. Liu, Q. Gao, Y. Gao, and Z. Feng, "Extreme self-paced learning machine for on-orbit sar images change detection," IEEE Access, vol. 7, pp. 116413-116423, Aug. 2019.

[14] F. Wang, Y. Wu, Q. Zhang, P. Zhang, M. Li, and Y. Lu, "Unsupervised change detection on SAR images using triplet markov field model," IEEE Geoscience and Remote Sensing Letters, vol. 10, no. 4, pp. 697701, Oct. 2013.

[15] M. Matsuoka and F. Yamazaki, "Use of satellite SAR intensity imagery for detecting building areas damaged due to earthquakes," Earthquake Spectra - EARTHQ SPECTRA, vol. 20, Aug. 2004.

[16] — , "Use of satellite sar intensity imagery for detecting building areas damaged due to earthquakes," Earthquake Spectra, vol. 20, no. 3, pp. 975-994, Aug. 2004.

[17] G. H. M. Voigt, D. I. Alves, C. Müller, R. Machado, V. T. Vu, and M. I. Pettersson, "Change detection method for intensity VHF wavelengthresolution SAR images," Submitted to SPIE Remote Sensing Symposium: Image and Signal Processing for Remote Sensing XXVII, May 2021.

[18] U. A. Force. (2021, May) The sensor data management system. [Online]. Available: https://www.sdms.afrl.af.mil/

[19] C. Oliver and S. Quegan, Understanding synthetic aperture radar images, 1st ed. SciTech Publishing, Jan. 2004.

[20] S. Nadarajah and S. Kotz, "Reliability for some bivariate exponential distributions," Mathematical Problems in Engineering, vol. 2006, pp. 1-14, Feb. 2006.

[21] D. I. Alves, B. G. Palm, H. Hellsten, V. T. Vu, M. I. Pettersson, R. Machado, B. F. Uchôa-Filho, and P. Dammert, "Wavelengthresolution SAR change detection using Bayes' theorem," IEEE Journal of Selected Topics in Applied Earth Observations and Remote Sensing, vol. 13, pp. 5560-5568, Sep. 2020. 